\title{
Dynamic Stereoscopic 3D Parameter Adjustments for Enhanced Depth Discrimination
}

\author{
Arun Kulshreshth \\ Department of Computer Science \\ University of Central Florida \\ Orlando, FL 32816, USA \\ arunkul@knights.ucf.edu
}

\author{
Joseph J. LaViola Jr. \\ Department of Computer Science \\ University of Central Florida \\ Orlando FL 32816, USA \\ jj1@eecs.ucf.edu
}

\begin{abstract}
Most modern stereoscopic 3D applications use fixed stereoscopic 3D parameters (separation and convergence) to render the scene on a 3D display. But, keeping these parameters fixed during usage does not always provide the best experience since it can reduce the amount of depth perception possible in some applications which have large variability in object distances. We developed two stereoscopic rendering techniques which actively vary the stereo parameters based on the scene content. Our first algorithm calculates a low resolution depth map of the scene and chooses ideal stereo parameters based on that depth map. Our second algorithm uses eye tracking data to get the gaze direction of the user and chooses ideal stereo parameters based on the distance of the gazed object. We evaluated our techniques in an experiment that uses three depth judgment tasks: depth ranking, relative depth judgment and path tracing. Our results indicate that variable stereo parameters provide enhanced depth discrimination compared to static parameters and were preferred by our participants over the traditional fixed parameter approach. We discuss our findings and possible implications on the design of future stereoscopic $3 \mathrm{D}$ applications.
\end{abstract}

\section{Author Keywords}

Stereoscopic 3D; Dynamic Stereo Parameters; Eye Tracking; Depth Judgment; ; Visual Fatigue; User Study; Video Games; User Experience; HCI.

\section{ACM Classification Keywords}

H.5.m Information Interfaces and Presentation (e.g. HCI): Miscellaneous.; K.8.0 Personal Computing: Games

\section{General Terms \\ Design, Experimentation, Measurement, Performance, \\ Human Factors.}

\section{INTRODUCTION}

Stereoscopic 3D displays present two images offset to the left and right eye of the user and these images are then fused by the brain to give the perception of $3 \mathrm{D}$ depth. The generation

Permission to make digital or hard copies of all or part of this work for personal or classroom use is granted without fee provided that copies are not made or distributed for profit or commercial advantage and that copies bear this notice and the full citation on the first page. Copyrights for components of this work owned by others than ACM must be honored. Abstracting with credit is permitted. To copy otherwise, or republish, to post on servers or to redistribute to lists, requires prior specific permission and/or a fee. Request permissions from permissions@ acm.org.

CHI'16, May 7 - 12, 2016, San Jose, CA, USA

(c) 2016 ACM $978-1-4503-3362-7 / 16 / 05 \ldots \$ 15.00$

http://dx.doi.org/10.1145/2858036.2858078

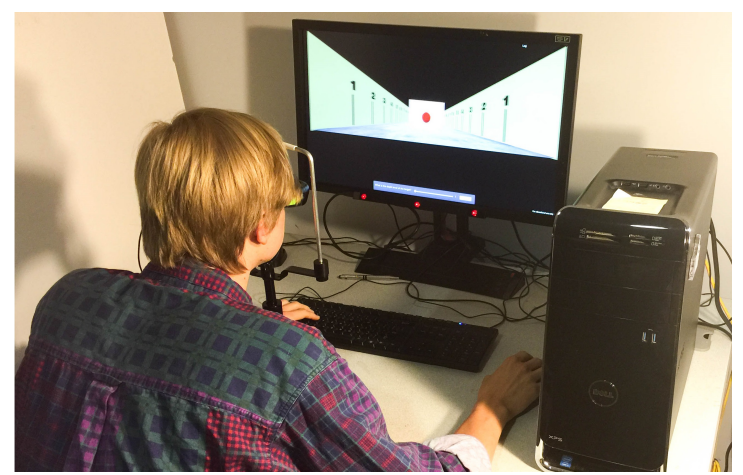

Figure 1. Dynamic stereoscopic parameters adjustment enhances depth discrimination ability. Ideal stereo parameters are selected based on the depth information of the scene.

of these two images uses two stereo parameters: separation and convergence. Separation is defined as the interaxial distance between the centers of the two virtual eye camera lenses in the scene and the convergence is defined as the distance of the plane where left and right eye camera frustums intersect (see Figure 2). Currently, most stereoscopic 3D applications (e.g. gaming, scientific/data visualizations, virtual reality experiences, etc.) fix convergence and separation values for comfortable viewing experience during usage. This approach is shown to be effective only for objects less than 25 meters away, and it works best for objects that are much closer (less than 10 meters) [23]. A larger value of separation and convergence is required to perceive depth for objects which are farther away. Traditionally, it has been believed that motion parallax and occlusion are more reliable depth cues than stereo for distances larger than 25 meters. However, we believe that dynamic stereo parameters adjustments could enhance depth discrimination ability even for larger distances.

In some 3D applications (e.g. first person shooter games) the distance of objects from the camera vary from scene to scene (e.g. moving from an indoor scene to outdoors) and using fixed parameters limits depth perception for such applications. In real life, we continuously adjust the distance between our eye pupils and converge to different distances based on the object we are looking at. Therefore, it is interesting to explore dynamic stereo parameter adjustment for 3D applications and see if it is possible to provide better depth perception regardless of object distance from the viewer.

The concept of dynamic stereo parameter was first proposed by Ware [23] where they explored the effects of dynamically 


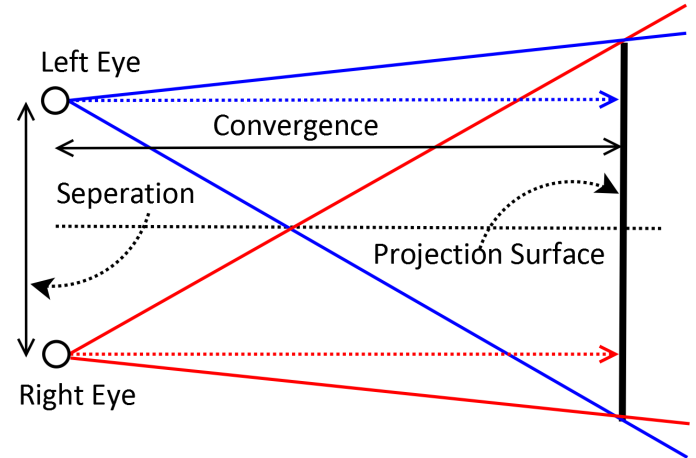

Figure 2. Off-Axis stereo projection.

adjusting separation value. Several researchers $[3,8,21]$ have explored this idea of stereo parameter adjustments for static images or videos. However, the previous approaches required offline processing and manual input in some cases [8]. We need to adjust stereo parameters in realtime in the case of 3D virtual environments such as video games. Ware et al. [25] explored dynamic adjustment of separation in realtime for 3D applications but no thorough user testing with depth judgment tasks was performed. Furthermore, it is also important that the stereo rendering is comfortable during usage and a user should not feel any scene distortion as the parameters change.

In this paper, we explore the usability of dynamic stereo parameter adjustments for 3D applications. We developed two dynamic stereo adjustment algorithms. The first algorithms selects ideal stereo parameters based on distance of the closest object and the second algorithm selects ideal stereo parameters based on the distance of the object looked at (obtained from eye tracking data). We conducted a within subjects experiment that uses three depth judgment tasks: depth ranking, relative depth judgment, and path tracing. We examined performance data (error magnitude for the depth ranking task and accuracy for the other two tasks), response time, and self-reported likert scale data on users' perceptions of the 3D scenes they looked at.

\section{RELATED WORK}

Recent work on stereoscopic 3D found it useful for games depending upon the task involved $[6,13,18]$. Researchers have also confirmed increased engagement and preference for stereoscopic 3D games [14, 18]. Stereoscopic 3D benefits can only be expected if the stereoscopic vision is not accompanied by distortions (e.g., contradicting depth cues, ghosting/cross-talk, exaggerated disparity) [26]. While stereoscopic 3D has shown some positive benefits depending on the task, it also has shown to cause negative symptoms as well, such as eyestrain, headache, dizziness, and nausea [10].

There are a few approaches to reduce visual fatigue in stereo rendered content. One approach to reduce visual fatigue is to use a smaller separation value [19]. This limits the rendered stereo images to be in a safe parallax range for visualization. However, this approach reduces the depth perception in the scene making the scene look almost like a 2D scene. Another approach [21] is to use non-linear image warping where a non-linear transfer function is applied to a disparity map and thus creates a depth distorted version of the original image that is visually comfortable. The main problem with this approach is that it is not realtime and is more suitable for postproduction of 3D movies. This method can not be applied to 3D applications which require realtime adjustment to produce visually comfortable 3D scenes.

Ware [23] proposed dynamic adjustment of the stereo separation parameter to minimize visual discomfort and increase stereo depth. Furthermore, the results revealed that the separation must be changed gradually over a few seconds to allow users to adjust without noticing any visual distortion of the scene. Our work optimizes both separation and convergence parameters for a better visual experience with enhanced depth discrimination. Ware et al. [25] explored dynamic adjustment of separation in realtime for 3D applications. Their results revealed that rate of separation should be kept below 0.2 $\mathrm{cm} / \mathrm{sec}$. However, their algorithm was not rigorously tested for depth judgment tasks and would not perform well when there is a object within a few feet from the camera. We used an eye tracking device to find the gaze direction of the user and selected ideal 3D parameters based on the object gazed. This enhances depth perception even in scenes with a object within a few feets of the camera.

Bernhard et al. [3] explored dynamic adjustment of stereo parameters using gaze data and found that it reduces stereo fusion time and provides a more comfortable viewing experience. Gurrieri [8] also used eye tracking data to adjust the stereo images for visual comfort. Their [8] approach used manually generated disparity maps which is not feasible for a realtime 3D viewing application. Kulshreshth et al. [12] explored dynamic stereo parameter adjustments for 3D applications. However, no formal user evaluation was conducted.

Several researchers $[3,5,20]$ have explored gaze based depth of field (DOF) effects to minimize visual fatigue. However, people generally disliked the DOF effect with temporal lag of the gaze-contingent effect being a possible reason. Maiello et al. [15] explored the interaction of stereo disparity parameters and DOF blur on stereo fusion. They found that DOF blur helped the stereo fusion process but only if the blur was present on the visual periphery. Mauderer et al. [16] used gaze contingent DOF to produce realistic 3D images and found that it increases the sense of realism and depth. But, their system had limited accuracy in terms of depth judgment of the objects in the scene.

The past work on dynamic stereo mentioned above used simple static scenes (e.g. random-dot stereograms, a picture, etc.) to evaluate their work. None of the work rigorously explored the benefits of dynamic stereo in 3D virtual environments requiring realtime adjustment of separation and convergence parameters. To the best of our knowledge, our work is the first to systematically explore dynamic stereo for 3D virtual environments using several depth judgment tasks.

\section{STEREOSCOPIC ALGORITHMS}

The main properties of the stereo algorithms are summarized in Table 1. These algorithms choose the 3D stereo parameters based on the scene displayed, display size, display aspect ratio, and the inter-pupilarity distance (IPD) of the user. We 


\begin{tabular}{|l|c|c|c|}
\hline Properties & Static Stereo & Dynamic Stereo & Dynamic Stereo with Eye Tracking \\
\hline Basis of 3D parameters change? & Fixed at start & The depth of the closest object. & The depth of the object the user is looking at. \\
\hline Closest object limits depth perception? & Yes & Yes & No \\
\hline Frequency of 3D parameter adjustment? & No Change & Lower & Higher \\
\hline $\begin{array}{l}\text { Chances of 3D parameter adjustment } \\
\text { detection by user? }\end{array}$ & N/A & Minimal & $\begin{array}{l}\text { Higher chance especially when the depth } \\
\text { range of the scene is large. }\end{array}$ \\
\hline
\end{tabular}

Table 1. Properties of the stereo algorithms.

defined a normalized maximum value for separation distance as

$$
\text { MaxSeparation }=\frac{\text { IPD }}{\text { Display Width }} \times \text { Scale Factor }
$$

where the Scale Factor is the scale factor to transform from the real world distances to the virtual world distances. Note that the IPD and the display width should have the same distance units in the above formula. In all the following stereo modes, the separation value is set to a percentage of this maximum separation value. The maximum separation value is based on Nvidia's recommendation for the best 3D experiences [1]. To give an example, for an average IPD of $65 \mathrm{~mm}$ (0.065 meters) [4], a 27 inch display with $16: 9$ aspect ratio (display width of 25.53 inches which is 0.648 meters), and scale factor of 10, the MaxSeparation is 1.002 .

\section{Static Stereo}

Static stereo uses fixed 3D stereo parameters which are chosen to provide a good 3D experience in all possible scenarios. As the distance of the objects from the user increase, the depth discrimination ability of the user decreases. Usually the parameters are chosen such that it reduces visual comfort even when the objects are too close. This reduces visual fatigue but the stereo effect is greatly reduced for farther objects. Using static parameters limits depth perception for farther objects when the depth range of the scene is very large (e.g., a FPS game with a gun very close to the camera and enemies at large distances). The stereo parameters are chosen such that the close object (e.g. gun in a FPS game) has minimal parallax for comfortable viewing. However, this limits depth discrimination ability for farther objects ( $>25$ meters).

\section{Dynamic Stereo}

This section describes the two dynamic stereo algorithms developed. The first algorithm selects ideal 3D parameters (separation and convergence) based on the depth of the closest object in the scene. The second algorithm selects ideal 3D parameters based on the depth of the object the user is looking at (obtained from eye tracking data). We conducted several pilot studies to determine ideal stereo parameters for different object distances. We considered object distance up to 250 meters away and divided this distance into several distance ranges (see Table 2). For each distance range, we tried several values for separation ranging from 0 to MaxSeparation (as defined in equation 1) with convergence values set to the distance of the object and chose the separation value that provided good depth perception with minimal visual fatigue.
Furthermore, we noticed that for objects farther than 200 meters, separation value set to MaxSeparation worked well and there was no change in stereo depth as we increased the convergence distance. Therefore, we choose separation value of MaxSeparation and convergence value of 200 meters for all objects farther than 200 meters. In addition, we also explored the rate at which these parameters have to be changed in order to allow enough time for a user to gradually adjust to new parameters. The values for separation, convergence, and rate of parameter change are summarized in Table 2. For all the objects farther than 10 meters, the parameters were changed at the rate of $15 \%$ of the change (the difference between the new value and the previous value). However, special consideration is given to objects less than 10 meters away since these objects can make stereo uncomfortable to view if rendered with large disparity. The parameters are changed at a higher rate for these close objects such that they are always comfortable to view in stereo.

\section{Dynamic Stereo (DS)}

This mode chooses ideal 3D parameters based on the distance of the closest object to the camera. In order to find the distance of the closest object, the scene is divided into several zones and the distance is calculated at the center of each zone using ray-casting. The number of zones depends on the size of the rendered frame and the size of the objects in the frame. In our case, we had a frame size of $1920 \times 1080$ pixels and we chose to divide it to $32 \times 16$ zones. Once the minimum distance is found the stereo separation is set based on the values mentioned in Table 2 and convergence distance is set to the minimum of the distance of the closest object and 200 meters. The current stereo parameters are gradually changed at a specified rate (see Table 2) to match the target parameters.

\section{Eye-Tracked Dynamic Stereo (EDS)}

This mode chooses ideal 3D parameters based on the distance of the object the user is currently looking at (obtained from eye tracking data). Once the minimum distance is found the stereo separation is set based on the values mentioned in Table 2 and convergence distance is set to the minimum of the distance of the gazed object and 200 meters. The current stereo parameters are gradually changed at a specified rate (see Table 2) to match the target parameters. When there is a close object within 4 meters of the camera, looking at farther objects causes a lot of disparity for that close object making stereo viewing uncomfortable. To improve visual discomfort, the close object was made partially transparent when a user is not looking at it. The color of that closed object is changed back to normal color when a user looks at it (see Figure 3). 


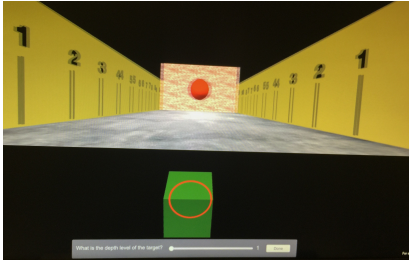

(a)

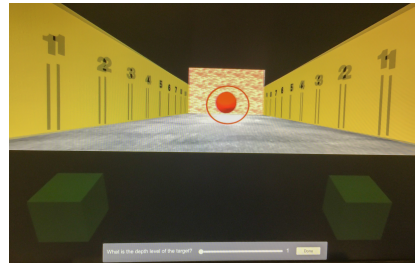

(b)
Figure 3. Eye-tracked dynamic stereo. The red circle indicates the gazed area. (a) When a close object is looked at it is in focus with its regular color (b) When a close object is not looked at it becomes partially transparent. Note that these images are stereo images taken from the display and that is why you see two images of the cube in the right image.

\begin{tabular}{|c|c|c|c|}
\hline Distance Range & Convergence & Separation & $\begin{array}{l}\text { Rate of } \\
\text { Parameter } \\
\text { Change (per } \\
\text { second) }\end{array}$ \\
\hline$<4$ meters & depth & $1 \%$ & $50 \%$ \\
\hline $4-7$ meters & depth & $5 \%$ & $25 \%$ \\
\hline $7-10$ meters & depth & $10 \%$ & $15 \%$ \\
\hline $10-25$ meters & depth & $20 \%$ & $5 \%$ \\
\hline $25-55$ meters & depth & $40 \%$ & $5 \%$ \\
\hline $55-80$ meters & depth & $60 \%$ & $5 \%$ \\
\hline $80-200$ meters & depth & $80 \%$ & $5 \%$ \\
\hline$>200$ meters & 200 meters & $100 \%$ & $5 \%$ \\
\hline
\end{tabular}

Table 2. Stereo parameters and rate of change for different distance ranges. Here depth is the distance of the closest object in the case of dynamic stereo and the distance of the object looked at in case of the eyetracked dynamic stereo. Separation is a percentage of MaxSeparation as defined in equation 1 and parameter change rate is a percentage of the total change desired.

\section{USER EVALUATIONS}

We conducted an experiment with our dynamic stereo algorithms to evaluate their effectiveness in terms of depth discrimination ability and response time of users. Since our dynamic algorithms rely on object distances, a close object could significantly affect performance in a depth judgment task. Therefore, in our experiment, we considered if there was a close object or not. Based on previous findings in related work and our analysis of the stereo algorithms, we have the following hypotheses:

Hypothesis 1 (H1) : Both dynamic stereo algorithms will improve depth discrimination ability in a 3D scene.

Hypothesis 2 (H2) : Dynamic stereo algorithms will reduce the response time of the users for depth judgment tasks compared to static stereo.

Hypothesis 3 (H3) : Eye-tracked dynamic stereo and dynamic stereo will provide similar depth discrimination abilities when there is no object within a few meters of the camera.

Hypothesis 4 (H4) : Eye-tracked dynamic stereo will perform better than dynamic stereo when there is a object within a few meters of the camera.

Hypothesis 5 (H5) : Users will prefer to use dynamic stereo over static stereo.

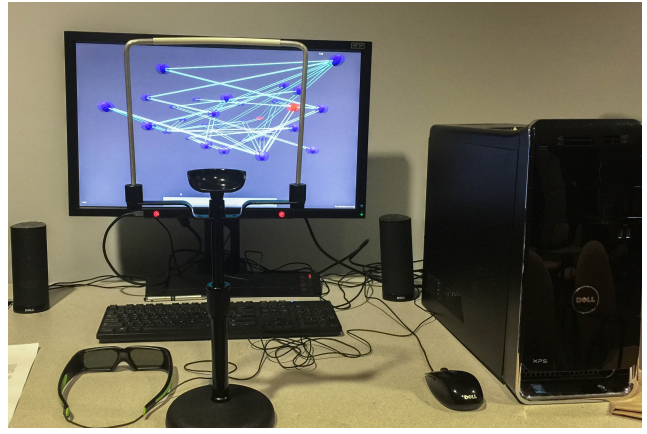

Figure 4. The Experimental Setup.

\section{Subjects and Apparatus}

We recruited 36 participants (27 males and 9 females ranging in age from 18 to 33 with a mean age 21.86) from the university population. We used an eye tracking device for this experiment which requires direct line of sight and using vision correction glasses along with 3D glasses would reduce the accuracy of the eye tracking data. Therefore, we selected participants which had either correct vision without glasses or corrected vision with contact lenses. We had 5 participants with contact lenses. Out of all participants, only 6 had prior experience with stereoscopic 3D games, and all the participant had prior experience of watching a 3D movie. The experiment duration ranged from 45 to 60 minutes, depending upon how fast participants finished the tasks, and all participants were paid $\$ 10$ for their time. Since our algorithms are customized for each person, we also measured the interpupilarity distance (IPD) of each user using a scale. The IPD ranged from $61 \mathrm{~mm}$ to $79 \mathrm{~mm}$ with mean value of $69.16 \mathrm{~mm}$ $(\sigma=4.21)$.

The experiment setup, shown in Figure 4, consisted of a 27" 3D monitor (BenQ XL2720Z), wired Nvidia 3D glasses, the Tobii EyeX eye tracker, a chinrest, and a PC (Core i7 4770K CPU, GTX 780 graphics card, 8 GB RAM). A mouse was used for answering questions during the experiment. The Tobii eye tracker was mounted at the bottom of the monitor. Participants were asked to place their chin on the chinrest during the experiment. The chinrest was centered on screen and was placed at a distance of 2 feet from the display screen. A chinrest was used to fix the user's position for collecting better eye tracking data for our eye-tracked dynamic stereo algorithm. In addition, a fixed position provides a better stereo viewing experience to a user by positioning them at the sweet spot for stereoscopic 3D. We used the Unity3D game engine and Nvidia's NVAPI for implementing the experimental tasks. To make sure that all our participants are able to see stereoscopic 3D, we used Nvidia's medical test image to test stereo abilities of the participants. All our participants passed the test.

\section{Experimental Tasks}

The participants were given three different depth judgment tasks: depth ranking, relative depth measurement, and path tracing. All our tasks were optimized for the best stereoscopic 3D experience:

- Stereoscopic 3D Specific GUI Elements: Our 2D GUI design was based on [17] for a better stereoscopic 3D expe- 


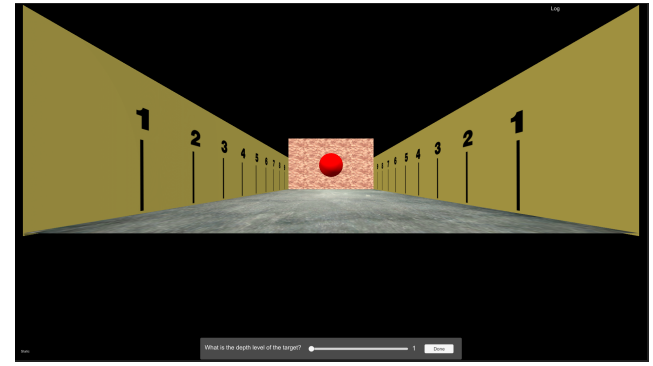

Figure 5. Depth ranking task with no close object. Participants ranked the depth of the sphere based on depth scale displayed on both side walls.

rience. All the 2D GUI elements were rendered at screen depth such that they are always in focus with no disparity.

- Disabled Monocular Cues: Shadows and specular reflections could also be used as depth cues. We disabled shadows for all the scene objects and all the scene lights were too far away (equivalent of Sunlight at infinity) to minimize specular reflections. In addition, our target objects had simple colors instead of textures. We wanted to avoid their influence in the depth judgment tasks.

- Minimized 3D Glasses Flicker: Initially, we started using Nvidia 3D wireless glasses. However, we noticed very severe flickering when used with the Tobii eye tracker due to interference of IR signals between the IR blaster on the eye tracker and the Nvidia's IR based 3D sync signal emitter. Therefore, we decided to use wired Nvidia 3D glasses and they worked fine with no issues.

\section{Depth Ranking Task}

This task has been used in prior research as a depth judgment task [7] and is very similar to tasks used to evaluate the effect of shadows on perception of depth and spatial relationships $[11,22]$. In this task, participants were asked to rank the depth of a sphere floating above the floor in a 3D graphical scene with uniformly distributed tick-marks (1 to 9), indicating depth levels, on the side walls (see Figure 5). Each tickmark was 25 meters apart with the first mark at 50 meters from the camera. The participants were asked to determine the depth of the sphere by indicating which tick-mark they thought it was aligned with. The depth of the sphere was randomly changed for each trial and was perfectly aligned with one of the nine tick-marks. Furthermore, to avoid using size as a depth cue, we also scaled the sphere such that it appears more or less of the same size on screen no matter what the depth value is. To input the perceived depth value, participants were asked to adjust a slider displayed at the bottom of the screen and then press the "Done" button displayed next to the slider. We recorded error magnitude and response time of the participants. The error magnitude was defined as the average absolute difference between the correct depth and judged depth over 10 trials and the response time was the average time over 10 trials taken from when the sphere appeared on screen to the time when they clicked the "Done" button.

\section{Relative Depth Judgment Task}

This task is a variation of the depth ranking task described above. Depth ranking tasks are more focused on measuring absolute depth of the target object. This task focuses on measuring the ability to judge relative depth of objects at differ-

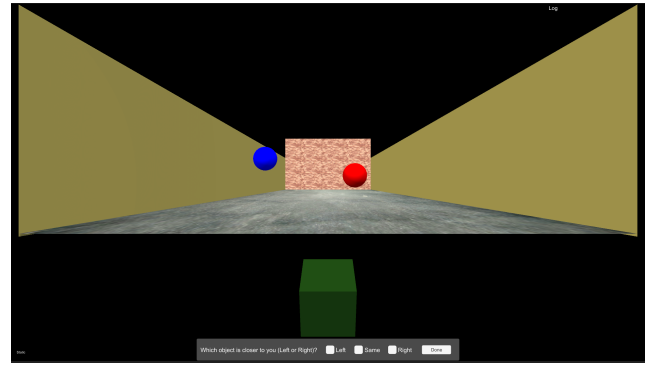

Figure 6. Relative depth judgment task with a close object at 3 meters from the camera. Participants determined which of the two spheres is closer to them. Possible answers were left, same, and right.

ent distances. In this task, two targets were displayed floating above the floor in a 3D graphical scene (the scene was the same as in the depth ranking task). The depth of the targets were randomly changed from 25 meters to 250 meters from the camera. The participants were asked to determine which of the two targets (left or right) is closer to them. The two targets were mentioned as left and right instead of blue and red to avoid any issues for color blind participants. The possible answers were "Left", "Right", and "Same" selected using toggles at the bottom of the screen. Immediately after selecting a toggle, participants were asked to press the "Done" button to go to the next trial. We recorded accuracy and response time of the participants. The accuracy was defined as the percentage of correct responses over 10 trials and the response time was the average time over 10 trials taken from when the two targets appeared on screen to the time when they clicked the "Done" button.

\section{Path Tracing Task}

Path tracing tasks have previously been used to evaluate various stereoscopic 3D modes (stereo, head coupled stereo, etc.) $[2,24]$, and to compare depth perception on various 3D displays [7]. The ability to trace paths in such graphs is an important aspect to understanding such information networks. Our implementation of the path tracing task was based on [7, 24]. A randomly generated graph with 30 nodes and 40 edges was displayed on screen. The nodes were randomly placed in a cubical volume $(120 \times 50 \times 200$ cubic meter $)$ to use screen space optimally along with a large depth range (200 meters). While generating the graph, the nodes were randomly positioned such that they did not occlude each other. The nodes were divided into three groups of 10 nodes each. Two of these groups were considered to be leaf nodes, while the third group was considered to be intermediate nodes. Each node of the first leaf node group was randomly connected to exactly two intermediate nodes. Each node of the second leaf node group was randomly connected to exactly two intermediate nodes with priority given to intermediate nodes with no edges to avoid any orphaned nodes. In each graph, the nodes were colored blue and two leaf nodes were randomly highlighted with red color. The task of the user was to determine whether or not there was a path of length two from one highlighted node to the other. Because the highlighted nodes were both leaf nodes, they could never be connected directly (see Figure 7). To indicate if there was a path or not, participants were asked to select one of the two toggles, labeled "Yes" and "No" displayed at the bottom of screen and then press 


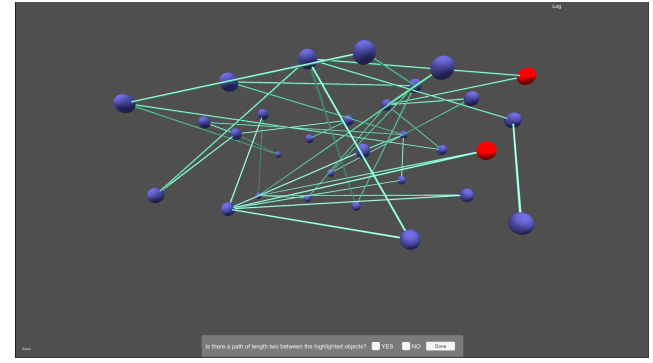

Figure 7. Path tracing task. Participants determined if there was a path of length two between the two highlighted red nodes.

the "Done" button displayed next to the toggles. We recorded accuracy and response time of the participants. The accuracy was defined as the percentage of correct responses over 10 trials and the response time was the average time over 10 trials taken from when the graph appeared on screen to the time when they clicked the "Done" button.

\section{Experiment Design and Procedure}

We chose a within-subjects design for our experiment in order to be able to measure and compare user performance and perceptions of the stereo algorithms on a variety of quantitative metrics. Each task had two independent variables: stereo mode (SM) (static stereo, dynamic stereo, dynamic stereo with eye tracking) and object mode (OM) (close object present and close object not present). In total we had $3 \times 2=6$ conditions for each task and for each conditions there were 10 trials which makes a total of $3 \times 6 \times 10=180$ trials for all the tasks. Our dependent variables were mean response time and task performance data (depth rank error for depth ranking task, and accuracy for the other two tasks).

The experiment began with the participant seated in front of the monitor and the moderator seated to the side. Participants were given a consent form that explained the experiment procedure. They were then given a pre-questionnaire which collected general information about the participant (age, gender, if they use contact lenses, and past experience with stereoscopic 3D games and movies). At the beginning, each participant was explained what they are supposed to do in each assigned task and were asked to perform each task a few times to make sure that they understood what they were supposed to do for each task. Participants performed the three tasks in order. For a given task, each condition $(6$ conditions per task) was presented to the user in preselected order based on a Latin square design. After each condition, the participant filled out a post-questionnaire with questions about their experiences with the stereo mode presented and if there were any negative symptoms (see Table 3 ).

\section{RESULTS}

To analyze the performance data of each task, we used repeated-measures 2-factor ANOVA per dependent variable. We did a post-hoc analysis using pairwise sample t-tests. We used Holm's sequential Bonferroni adjustment to correct for type I errors [9] and the Shapiro-Wilk test to make sure the data was parametric. To analyze the Likert scale data, we used Friedman's test and then a post-hoc analysis was done

\begin{tabular}{|c|l|}
\hline \multicolumn{2}{|c|}{ Post-Questionnaire } \\
\hline Q1 & $\begin{array}{l}\text { To what extent did you feel that the scene presented was in stereo- } \\
\text { scopic 3D? }\end{array}$ \\
\hline Q2 & To what extent is depth more defined and clearer? \\
\hline Q3 & $\begin{array}{l}\text { To what extent does it feel more like looking at real objects than } \\
\text { a picture of objects? }\end{array}$ \\
\hline Q4 & $\begin{array}{l}\text { To what extent did you notice double images of the same object } \\
\text { (ghosting)? }\end{array}$ \\
\hline Q5 & To what extent did objects appear distorted? \\
\hline Q6 & To what extent did objects appear transparent and translucent? \\
\hline Q7 & To what extent do you prefer this stereoscopic 3D mode? \\
\hline Q8 & $\begin{array}{l}\text { Did you feel any Symptoms from viewing the games in stereo- } \\
\text { scopic 3D (eye strain, headaches, dizziness, Nausea)? }\end{array}$ \\
\hline
\end{tabular}

Table 3. Post-Questionnaire. Participants responded to question 1-76 on a 7 point Likert scale. In question 8 , each symptom had a 7 point Likert scale to indicate the extent of each symptom ranging from not at all to very much so.

\begin{tabular}{|c|c|c|}
\hline Source & Error Magnitude & Response Time \\
\hline $\mathrm{OM}$ & $F_{1,35}=39.092, p<0.005$ & $F_{1,35}=1.937, p=0.173$ \\
\hline $\mathrm{SM}$ & $F_{2,34}=45.563, p<0.005$ & $F_{2,34}=0.050, p=0.951$ \\
\hline $\mathrm{OM} \times \mathrm{SM}$ & $F_{2,34}=21.127, p<0.005$ & $F_{2,34}=2.386, p=0.107$ \\
\hline
\end{tabular}

Table 4. Repeated measures 2-factor ANOVA results for depth ranking task. SM: Stereo Mode, OM: Object mode

using Wilcoxon signed rank test. For all of our statistical measures, we used $\alpha=0.05$.

\section{Depth Ranking Task}

Repeated measures 2-factor ANOVA results are shown in Table 4 . In terms of error magnitude, we found significant differences based on stereo mode, object mode, and their combination. Error magnitude was significantly higher for static stereo compared to dynamic stereo $\left(t_{35}=7.106, p<0.005\right)$ and eye-tracked dynamic stereo $\left(t_{35}=9.679, p<0.005\right)$. Dynamic stereo had significantly higher $\left(t_{35}=4.688, p<\right.$ $0.005)$ error magnitude compared to eye-tracked dynamic stereo. Error magnitude was significantly higher $\left(t_{35}=\right.$ $-6.252, p<0.005$ ) when there was a close object in front of the camera. Dynamic stereo with no close object had significantly less error magnitude $\left(t_{35}=-9.240, p<0.005\right)$ compared to dynamic stereo with a close object. We did not find any significant differences in response time based on stereo mode or object mode. See Figure 8 for average values of error magnitude and response time.

When broken down based on object mode, we found differences in error magnitude between the stereo modes (see Figure 9). In the case of no close object, static stereo had higher error magnitude compared to dynamic stereo $\left(t_{35}=8.445, p<0.005\right)$ and eye-tracked dynamic stereo $\left(t_{35}=8.749, p<0.005\right)$. There was no significant difference in error magnitudes between dynamic stereo and eyetracked dynamic stereo. When the close object was present, there was no significant difference in error magnitude between static stereo and dynamic stereo. Eye-tracked dynamic stereo had significantly less error magnitude compared to static stereo $\left(t_{35}=7.787, p<0.005\right)$ and dynamic stereo $\left(t_{35}=6.227, p<0.005\right)$. 


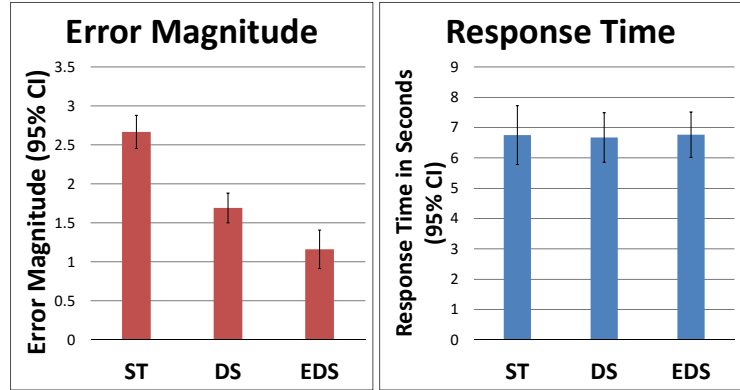

Figure 8. Error magnitude and response time of each stereo mode for depth ranking task where ST is static stereo, DS is dynamic stereo and EDS is eye-tracked dynamic stereo.

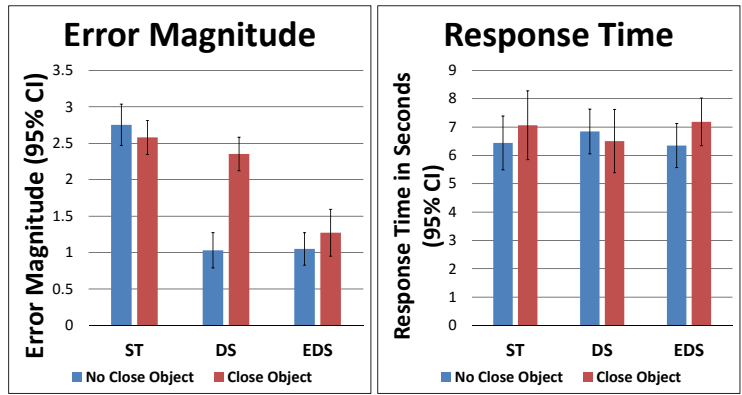

Figure 9. Error magnitude and response time for each object mode in case of depth ranking task where ST is static stereo, DS is dynamic stereo and EDS is eye-tracked dynamic stereo.

\section{Relative Depth Judgment Task}

Repeated measures 2-factor ANOVA results are shown in Table 5. In terms of accuracy, we found significant differences based on stereo mode, object mode, and their combination (see Figure 10). Static stereo had significantly less accuracy compared to dynamic stereo $\left(t_{35}=-5.999, p<0.005\right)$ and eye tracked dynamic stereo $\left(t_{35}=-10.029, p<0.005\right)$. Eye-tracked dynamic stereo was significantly more accurate than dynamic stereo $\left(t_{35}=-7.463, p<0.005\right)$. Accuracy was significantly higher $\left(t_{35}=6.015, p<0.005\right)$ when there was no close object present. No significant difference in response time was found based on stereo mode or object mode (see Figure 10). However, the combined interaction between stereo mode and object mode was significant. Dynamic stereo with no close object was significantly more accurate $\left(t_{35}=9.084, p<0.005\right)$ and had significantly less response time $\left(t_{35}=-2.864, p<0.01\right)$ when compared to dynamic stereo with a close object.

There were significant differences in accuracy and response time when we looked at each object mode separately (see Figure 11). In case of no close object, static stereo was significantly less accurate than dynamic stereo $\left(t_{35}=-8.980, p<\right.$ $0.005)$ and eye tracked dynamic stereo $\left(t_{35}=-7.834, p<\right.$ $0.005)$. Response time for dynamic stereo was significantly less $\left(t_{35}=-2.461, p<0.025\right)$ than eye-tracked dynamic stereo. When a close object was present, the accuracy of static stereo and dynamic stereo was not significantly different. Eye-tracked dynamic stereo was significantly more accurate than static stereo $\left(t_{35}=-8.859, p<0.005\right)$ and dynamic stereo $\left(t_{35}=-9.690, p<0.005\right)$.

\begin{tabular}{|c|c|c|}
\hline Source & Accuracy & Response Time \\
\hline $\mathrm{OM}$ & $F_{1,35}=36.181, p<0.005$ & $F_{1,35}=0.308, p=0.582$ \\
\hline $\mathrm{SM}$ & $F_{2,34}=50.923, p<0.005$ & $F_{2,34}=2.723, p=0.080$ \\
\hline $\mathrm{OM} \times \mathrm{SM}$ & $F_{2,34}=27.774, p<0.005$ & $F_{2,34}=4.658, p<0.05$ \\
\hline
\end{tabular}

Table 5. Repeated measures 2-factor ANOVA results for relative depth judgment task. SM: Stereo Mode, OM: Object mode

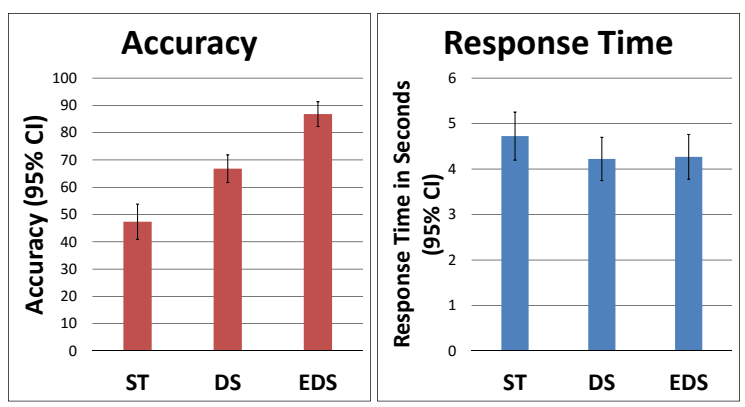

Figure 10. Accuracy and response time of each stereo mode for relative depth judgment task where ST is static stereo, DS is dynamic stereo and EDS is eye-tracked dynamic stereo.

\section{Path Tracing Task}

Repeated measures 2-factor ANOVA results are shown in Table 6. Accuracy had significant interaction based on stereo mode (see Figure 12). Static stereo was significantly less accurate than dynamic stereo $\left(t_{35}=-3.146, p<0.005\right)$ and eye-tracked dynamic stereo $\left(t_{35}=-4.745, p<0.005\right)$. Response time had significant differences based on object mode, stereo mode, and their combination (see Figure 12). Response time was significantly less $\left(t_{35}=-3.466, p<0.005\right)$ when no close object was present compared to when a close object was present. Eye-tracked dynamic stereo had significantly less response time $\left(t_{35}=2.519, p<0.02\right)$ compared to static stereo. Dynamic stereo with no close object had significantly less $\left(t_{35}=-4.371, p<0.005\right)$ response time compared to dynamic stereo with a close object. Eye-tracked stereo with no close object had significantly less $\left(t_{35}=-3.725, p<0.005\right)$ response time compared to eyetracked stereo with a close object.

We found significant differences in accuracy and response time when each object mode was analyzed separately (see Figure 13). In case of no close object, static stereo was significantly less accurate than dynamic stereo $\left(t_{35}=-3.820, p<\right.$ $0.005)$ and eye-tracked dynamic stereo $\left(t_{35}=-5.012, p<\right.$ $0.005)$. Static stereo had significantly more response time than dynamic stereo $\left(t_{35}=3.163, p<0.005\right)$ and eyetracked dynamic stereo $\left(t_{35}=3.454, p<0.005\right)$ when no close object was present. When a close object was present, eye-tracked dynamic stereo was significantly more accurate than static stereo $\left(t_{35}=-2.462, p<0.02\right)$. No significant difference in response time was found between the stereo modes when a close object was present.

\section{Post-Questionnaire Data}

For likert scale data, an average over the three tasks (depth ranking, relative depth judgment, and path tracing) was calculated and then analyzed using Friedman's test. The results for questions 1-7 are summarized in Table 7 . There were significant differences for all the questions. Mean ratings for questions 1 to 7 (see Table 3) are summarized in Figure 14. 


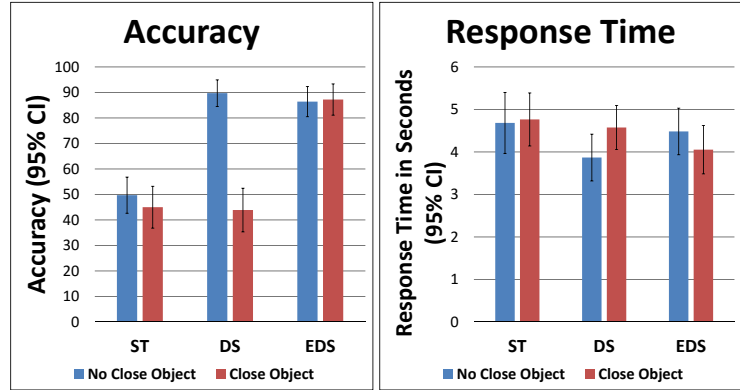

Figure 11. Accuracy and response time of each object mode for relative depth judgment task where ST is static stereo, DS is dynamic stereo and EDS is eye-tracked dynamic stereo.

\begin{tabular}{|c|c|c|}
\hline Source & Accuracy & Response Time \\
\hline $\mathrm{OM}$ & $F_{1,35}=1.272, p=0.267$ & $F_{1,35}=12.015, p<0.005$ \\
\hline $\mathrm{SM}$ & $F_{2,34}=11.275, p<0.005$ & $F_{2,34}=3.368, p<0.05$ \\
\hline $\mathrm{OM} \times \mathrm{SM}$ & $F_{2,34}=1.453, p=0.248$ & $F_{2,34}=4.061, p<0.05$ \\
\hline
\end{tabular}

Table 6. Repeated measures 2-factor ANOVA results for path tracing task. SM: Stereo Mode, OM: Object mode

Question 1 (Stereo Perception): In case of no close object, dynamic stereo $(Z=-5.164, p<0.005)$ and eye-tracked dynamic stereo $(Z=-5.212, p<0.005)$ provided significantly more stereo perception compared to static stereo. In case of the close object, eye-tracked dynamic stereo provided significantly more stereo perception compared to dynamic stereo $(Z=-4.990, p<0.005)$ and static stereo $(Z=-4.984, p<0.005)$. Dynamic stereo with no-close object provided significantly more $(Z=-5.003, p<0.005)$ stereo perception compared to dynamic stereo with a close object.

Question 2 (Depth Perception): In case of no close object, dynamic stereo $(Z=-5.162, p<0.005)$ and eye-tracked dynamic stereo $(Z=-5.021, p<0.005)$ provided significantly more depth perception compared to static stereo. In case of the close object, eye-tracked dynamic stereo provided significantly more depth perception compared to dynamic stereo $(Z=-4.892, p<0.005)$ and static stereo $(Z=-4.720, p<0.005)$. Dynamic stereo with no-close object provided significantly more $(Z=-5.134, p<0.005)$ depth perception compared to dynamic stereo with a close object.

Question 3 (Realism): In case of no close object, dynamic stereo $(Z=-4.887, p<0.005)$ and eye-tracked dynamic stereo $(Z=-4.456, p<0.005)$ provided significantly more realism compared to static stereo. In case of the close object, eye-tracked dynamic stereo provided significantly more depth perception compared to dynamic stereo $(Z=-4.709, p<$ $0.005)$ and static stereo $(Z=-4.589, p<0.005)$. Dynamic stereo with no-close object provided significantly more $(Z=-4.908, p<0.005)$ realism compared to dynamic stereo with a close object.

Question 4 (Ghosting): Eye-tracked dynamic stereo with a close object had significantly more ghosting compared to the eye-tracked dynamic stereo with no close object $(Z=$ $-3.721, p<0.005)$, static stereo with close object $(Z=$ $-4.137, p<0.005)$, and dynamic with a close object $(Z=$

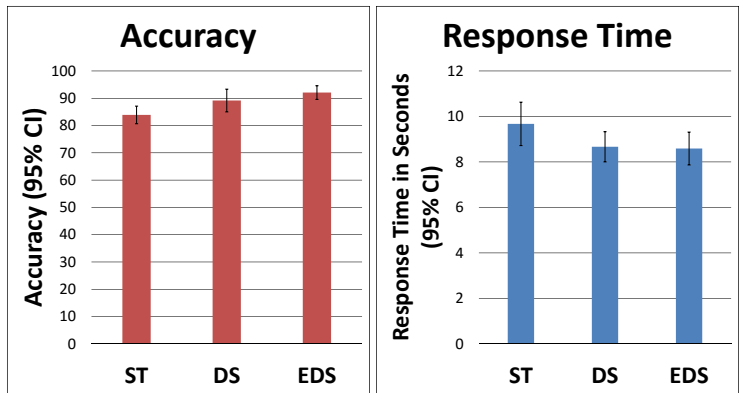

Figure 12. Accuracy and response time of each stereo mode for path tracing task where ST is static stereo, DS is dynamic stereo and EDS is eye-tracked dynamic stereo.

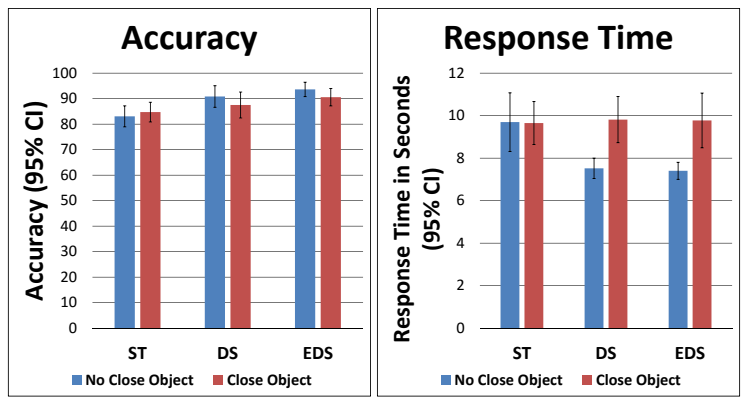

Figure 13. Accuracy and response time of each object mode for path tracing task where ST is static stereo, DS is dynamic stereo and EDS is eye-tracked dynamic stereo.

$-3.617, p<0.005)$. Although, the ghosting was significantly higher for eye-tracked dynamic stereo with close object but the mean rating was still low with a value of 2.37 on a 7 point likert scale.

Question 5 (Distortion): Participants reported that eyetracked dynamic stereo with a close object had significantly more distortions compared to eye-tracked with no close object $(Z=-2.976, p<0.005)$, static stereo with a close object $(Z=-3.393, p<0.005)$, and dynamic with a close object $(Z=-2.445, p<0.025)$. Although, the distortion was significantly higher for eye-tracked dynamic stereo with a close object, this rating was still acceptable with a mean value of 1.77 on a 7 point likert scale.

Question 6 (Transparency): Participants reported that eyetracked dynamic stereo with a close object had significantly more transparency compared to eye-tracked dynamic stereo with no close object $(Z=-2.492, p<0.025)$, and dynamic stereo with a close object $(Z=-2.473, p<0.025)$. Although, the distortion was significantly higher for eye-tracked with a close object, the rating was still acceptable with value of 1.50 on a 7 point likert scale.

Question 7 (Preference): Participants preferred both dynamic stereo $(Z=-4.944, p<0.005)$ as well as eyetracked dynamic stereo $(Z=-4.984, p<0.005)$ over static stereo when no close object was present. Eye-tracked dynamic stereo was preferred over static $(Z=-4.166, p<$ $0.005)$ and dynamic stereo $(Z=-4.140, p<0.005)$ when a close object was present.

Except for minor eye strain, none of the participants, except two, noticed any symptoms (headache, dizziness, or nausea) 


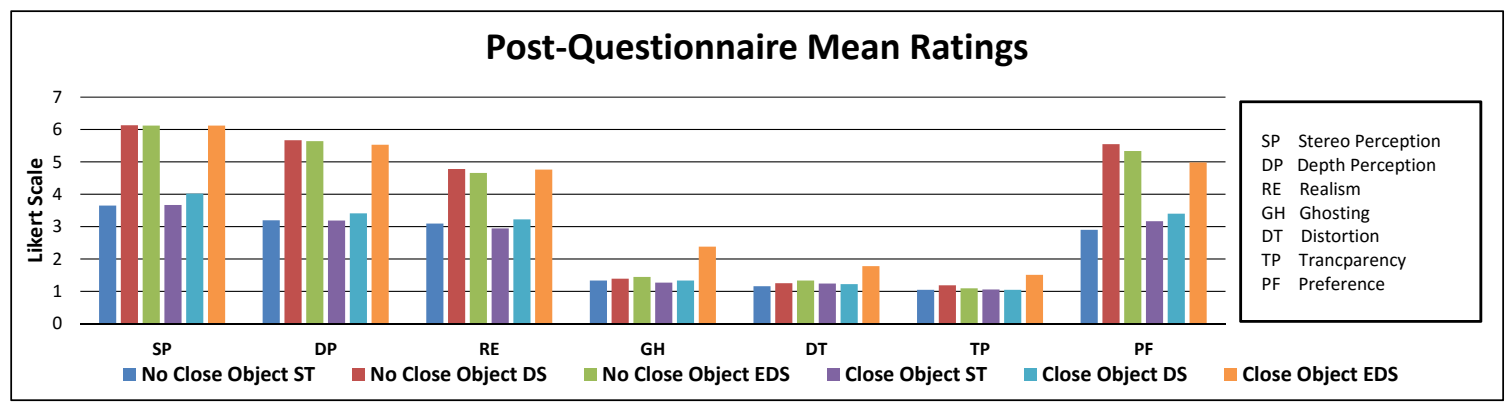

Figure 14. Mean ratings for post-questionnaire questions where ST is static stereo, DS is dynamic stereo, and EDS is eye-tracked dynamic stereo.

\begin{tabular}{|c|c|}
\hline Question & Friedman's test \\
\hline Q1 (SP) & $\chi^{2}(5)=125.178, p<0.0005$ \\
\hline Q2 (DP) & $\chi^{2}(5)=109.428, p<0.0005$ \\
\hline Q3 (RE) & $\chi^{2}(5)=89.285, p<0.0005$ \\
\hline Q4 (GH) & $\chi^{2}(5)=39.255, p<0.0005$ \\
\hline Q5 (DT) & $\chi^{2}(5)=32.772, p<0.0005$ \\
\hline Q6 (TP) & $\chi^{2}(5)=11.399, p<0.05$ \\
\hline Q7 (PF) & $\chi^{2}(5)=96.247, p<0.0005$ \\
\hline
\end{tabular}

Table 7. Results of Friedman's test for post-questionnaire likert scale data.

from viewing the scenes in stereoscopic 3D. Two of our participants were very sensitive to stereoscopic 3D and experienced minor headache by the end of the experiment.

\section{DISCUSSION}

We found significant performance differences between different stereo modes. Average error magnitude was significantly lower for both dynamic stereo algorithms in case of the depth ranking task. Average accuracy was significantly higher for both dynamic stereo modes in the case of the relative depth judgment task and the path tracing task. Both our dynamic stereo algorithms chose ideal stereo parameters for objects at different depths leading to better depth discrimination ability even at farther distances. Consequently, we were able to accept our first hypothesis H1.

Significant differences between the task response time were found based on the stereo mode for the path tracing task. In the case of the depth ranking task and the relative depth judgment task, the tasks were very simple and required a user to focus on the target objects to judge their depth. Therefore, the response times in all the stereo modes were very similar. In the case of the path tracing task, the scene was more complex requiring a thorough analysis of the scene to determine if there was a path of length two between the highlighted node or not. Thus, the response time for the path tracing task was higher than the response times for the other two tasks. In addition, the response time for the path tracing task was significantly lower for both dynamic stereo modes compared to static stereo. Based on all these findings, we were not able to accept our second hypothesis $\mathrm{H} 2$.

We found some significant differences based on the combined effect of stereo mode and object mode. In case of the depth ranking task and relative depth judgment task, the performance of non-eye-tracked dynamic stereo was significantly reduced when a close object was present compared to when the close object was absent. The presence of a close object limits depth perception for our dynamic stereo algorithm. The dynamic stereo algorithm calculates ideal stereo parameters based on the distance of the closest object. Therefore, the presence of an object within a few meters of the camera leads to a very low value of separation and convergence thereby limiting depth perception. For the depth ranking task and the relative depth task, the judgment of depth is very critical. Thus, the performance is greatly reduced when a close object is present. In the case of the path tracing task, judgment of depth helps to perform the task faster but is not critical to the task. One can still trace a path without stereo with good accuracy but it will take longer than when stereo is present. Therefore, we did not see any change in accuracy but higher response time when a close object was present for this task.

When we looked at each object mode separately, there were some interesting findings. For all the tasks, the performance of dynamic stereo and eye-tracked dynamic stereo was very close when there was no close object. Therefore, we were able to accept our third hypothesis (H3). However, the performance of dynamic stereo was very close to static stereo when a close object was present. As we mentioned earlier, the presence of a close object limits depth perception of our dynamic stereo algorithm and it produces stereo images which are very similar to that of a static stereo with a close object. However, our eye-tracked dynamic stereo could adjust parameters based on a user's gaze direction. Its performance is not affected by the presence of a close object. Consequently, we were able to accept our third and fourth hypothesis (H3 and $\mathrm{H} 4)$.

Surprisingly, in case of the path tracing task, the response time was not significantly different for the eye-tracked dynamic stereo as we expected. The main reason was the fact that the nearby object was rendered very close to the nodes in screen space (but never occluding any node) and when a user is looking at the nodes (which are much farther away than the close object), it leads to a large disparity for the close object. In case of the eye-tracked dynamic stereo, we tried to mitigate the effects of large disparity for a close object by making it partially transparent when the user is not looking at it. It worked quite well for the depth ranking task and the relative depth judgment task because the targets were away from the close object in screen space. However, it was distracting in case of path tracing task due to its vicinity from the rendered nodes in screen space, leading to more response time than expected. 
Post-questionnaire data revealed that participants preferred dynamic stereo over static stereo. As we expected, participants rated both dynamic algorithms to have higher stereo perception and depth perception when no close object was present. When a close object was present, eye-tracked stereo was rated to have higher stereo perception and depth perception. Participants preferred both dynamic stereo algorithms when there was no close object present. Participants preferred eye-tracked dynamic stereo over the other two stereo modes when a close object was present. Based on these findings, we were not able to accept our fifth hypothesis H5. For all the tasks, the scenes presented were optimized for a better $3 \mathrm{D}$ viewing experience and the total exposure time was short (within 60 minutes). Consequently, participants did not feel any negative symptoms from viewing the scenes in stereoscopic 3D.

Our experiments did not include any test cases to see how our algorithm will behave when a close object suddenly appears or disappears. However, our algorithms could be easily modified to handle such cases. When a close object suddenly appears, the stereo parameters should be changed (at a fast rate to avoid discomfort) such that the close object is in focus. When a close object suddenly disappears, the parameters should be gradually changed based on the depth of the remaining objects in the scene.

Our results seem promising for future stereoscopic 3D applications (especially for video games). In scenarios where eye tracking is not available, dynamic stereo could be utilized to choose ideal stereo parameters for video games which don't have any close objects (e.g. strategy games). Eye-tracked dynamic stereo could be very useful for first person shooter (FPS) games. FPS games have a close object (e.g., a gun) in front of the camera and this limits depth perception for farther enemies. Eye-tracked dynamic stereo could help increase depth perception when the user is not looking at the close object.

There are a few factors that could have affected our results. The values of the stereo parameters (see Table 2 ) for different distances were experimentally estimated using several pilot studies. These values worked for our experimental setup but other values in the vicinity could also be used and may lead to somewhat different results. However, we believe that the affect would be minor. The stereo display we used was optimized for usage with Nvidia 3D vision glasses and produced comfortable 3D images with minimal ghosting. Our dynamic stereo algorithms may not work for a stereo display with more ghosting. In addition, our algorithms were optimized for 3D viewing from a close distance and different stereo parameters may be required for dynamic stereo on a setup with a huge display and a larger viewing distance. Most participants in our user study were males and this gender imbalance could have a minor effect on our results. We would also like to mention that the use of dynamic stereo would change the geometry of the scene (e.g. an increase in separation makes the world seem smaller and/or the observer feel larger) and may not be a good idea in situations where scale is of critical importance such as in case of industrial design applications. Moreover, our experiment was controlled in order to study the effects of only stereo mode changes and practitioners should be aware of this fact before using our results directly for any stereoscopic application.

\section{CONCLUSION AND FUTURE WORK}

We presented an in-depth study which explored dynamic stereo parameter adjustments for enhanced depth discrimination ability. We presented two stereo rendering algorithms to dynamically adjust the stereo parameters (separation and convergence). The first algorithms selects ideal stereo parameters based on depth of the closest object and the second algorithm selects ideal stereo parameters based on the depth of the object looked at (obtained from eye tracking data). A within subjects experiment was conducted that used three depth judgment tasks: depth ranking, relative depth judgment, and path tracing, to evaluate the performance of our algorithms. Our results show that dynamic stereo parameters provide enhanced depth discrimination compared to static parameters and were preferred by our participants over the traditional fixed parameter approach. Furthermore, we found that the dynamic stereo algorithm without eye-tracking performed similarly to the static stereo with fixed parameters when there was a object within 4 meters of the camera. However, eyetracked dynamic stereo had similar performance in both cases with or without a close object in the vicinity of the camera.

In our experiment, we estimated the rate of stereo parameter change based on some pilot studies. Although, it was a rough estimate, it worked quite well for our experiments but a thorough study to calculate the best parameter change rate is still required. We would like to explore this in the future. We fixed the position of the user in our test scenes. In the future, we would like to explore dynamic stereo algorithms for video games or 3D applications where a user is allowed to move in the 3D virtual environment. Dynamic stereo might interact with other depth/perceptual cues and would be an interesting research direction for future. We used transparency to avoid any discomfort caused by a closer object when the user is looking at farther objects. Although this affect is artificial but it worked quite well for our purpose. In real world, our eyes blur closer objects when we are focusing on farther objects. Therefore, depth of field blurring would be an ideal choice instead of transparency. It will be interesting to explore this direction in the future.

\section{ACKNOWLEDGMENTS}

This work is supported in part by NSF CAREER award IIS0845921. We would like to thank Stephen M. Fiore for helping us design our user study. We would also like to thank the other members of ISUE lab for their support and the anonymous reviewers for their useful comments and feedback.

\section{REFERENCES}

1. Nvidia's GTC 2010 presentation. http://www . nvidia. com/content/GTC-2010/pdfs/2010_GTC2010.pdf, 2010 .

2. Arthur, K. W., Booth, K. S., and Ware, C. Evaluating 3D task performance for fish tank virtual worlds. ACM Transactions on Information Systems 11,3 (1993), 239-265. 
3. Bernhard, M., Dell'mour, C., Hecher, M., Stavrakis, E., and Wimmer, M. The effects of fast disparity adjustment in gaze-controlled stereoscopic applications. In Proceedings of the Symposium on Eye Tracking Research and Applications, ETRA '14, ACM (New York, NY, USA, 2014), 111-118.

4. Dodgson, N. A. Variation and extrema of human interpupillary distance, 2004.

5. Duchowski, A. T., House, D. H., Gestring, J., Wang, R. I., Krejtz, K., Krejtz, I., Mantiuk, R., and Bazyluk, B. Reducing visual discomfort of $3 \mathrm{~d}$ stereoscopic displays with gaze-contingent depth-of-field. In Proceedings of the ACM Symposium on Applied Perception, SAP '14, ACM (New York, NY, USA, 2014), 39-46.

6. Fujimoto, M., and Ishibashi, Y. The effect of stereoscopic viewing of a virtual space on a networked game using haptic media. In Proceedings of the ACM SIGCHI international Conference on Advances in Computer Entertainment, ACM (New York, NY, 2005), 317-320.

7. Grossman, T., and Balakrishnan, R. An evaluation of depth perception on volumetric displays. In Proceedings of the Working Conference on Advanced Visual Interfaces, AVI '06, ACM (New York, NY, USA, 2006), 193-200.

8. Gurrieri, L. Improvements in the visualization of stereoscopic 3d imagery. 3D Research 6, 3 (2015).

9. Holm, S. A simple sequentially rejective multiple test procedure. Scandinavian Journal of Statistics 6, 2 (1979), 65-70.

10. Howarth, P. A. Potential hazards of viewing 3D stereoscopic television, cinema and computer games: a review. Ophthalmic \& Physiological Optics : The Journal of the British College of Ophthalmic Opticians (Optometrists) 31, 2 (2011), 111-122.

11. Hubona, G. S., Wheeler, P. N., Shirah, G. W., and Brandt, M. The relative contributions of stereo, lighting, and background scenes in promoting 3D depth visualization. ACM Transactions on Computer-Human Interaction 6,3 (1999), 214-242.

12. Kulshreshth, A., and LaViola, Jr., J. J. Enhanced depth discrimination using dynamic stereoscopic $3 \mathrm{~d}$ parameters. In Proceedings of the ACM Conference Extended Abstracts on Human Factors in Computing Systems, CHI '15, ACM (New York, NY, USA, 2015), $1615-1620$.

13. Kulshreshth, A., Schild, J., and LaViola Jr., J. J. Evaluating user performance in 3D stereo and motion enabled video games. In Proceedings of the International Conference on the Foundations of Digital Games, ACM (New York, NY, 2012), 33-40.

14. Litwiller, T., and LaViola Jr., J. J. Evaluating the benefits of 3D stereo in modern video games. In Proceedings of the SIGCHI Conference on Human Factors in Computing Systems, CHI'11, ACM (New York, NY, USA, 2011), 2345-2354.
15. Maiello, G., Chessa, M., Solari, F., and Bex, P. J. Simulated disparity and peripheral blur interact during binocular fusion. Journal of vision 14, 8 (2014), 13.

16. Mauderer, M., Conte, S., Nacenta, M. A., and Vishwanath, D. Depth perception with gaze-contingent depth of field. In Proceedings of the SIGCHI Conference on Human Factors in Computing Systems, CHI '14, ACM (New York, NY, USA, 2014), 217-226.

17. Schild, J., Bölicke, L., LaViola Jr., J. J., and Masuch, M. Creating and analyzing stereoscopic 3D graphical user interfaces in digital games. In Proceedings of the SIGCHI Conference on Human Factors in Computing Systems, CHI '13, ACM (New York, NY, USA, 2013), $169-178$.

18. Schild, J., LaViola Jr., J. J., and Masuch, M. Understanding user experience in stereoscopic 3D games. In Proceedings of the SIGCHI Conference on Human Factors in Computing Systems, CHI '12, ACM (New York, NY, USA, 2012), 89-98.

19. Siegel, M., Tobinaga, Y., and Akiya, T. Kinder, gentler stereo. In Electronic Imaging'99, International Society for Optics and Photonics (1999), 18-27.

20. Vinnikov, M., and Allison, R. S. Gaze-contingent depth of field in realistic scenes: The user experience. In Proceedings of the Symposium on Eye Tracking Research and Applications, ETRA '14, ACM (New York, NY, USA, 2014), 119-126.

21. Wang, O., Lang, M., Stefanoski, N., Sorkine-Hornung, A., Sorkine-Hornung, O., Smolic, A., and Gross, M. Image Domain Warping for Stereoscopic 3D Applications. John Wiley \& Sons, Ltd, 2013, 207-230.

22. Wanger, L. C., Ferwerda, J. A., and Greenberg, D. P. Perceiving spatial relationships in computer-generated images. IEEE Computer Graphics and Applications, 3 (1992), 44-51.

23. Ware, C. Dynamic stereo displays. In Proceedings of the SIGCHI Conference on Human Factors in Computing Systems, CHI '95, ACM Press/Addison-Wesley Publishing Co. (New York, NY, USA, 1995), 310-316.

24. Ware, C., and Franck, G. Evaluating stereo and motion cues for visualizing information nets in three dimensions. ACM Transactions on Graphics 15, 2 (April 1996), 121-140.

25. Ware, C., Gobrecht, C., and Paton, M. Dynamic adjustment of stereo display parameters. Systems, Man and Cybernetics, Part A: Systems and Humans, IEEE Transactions on 28, 1 (Jan 1998), 56-65.

26. You, J., Jiang, G., Xing, L., and Perkis, A. Quality of visual experience for 3D presentation - stereoscopic image. In High-Quality Visual Experience, M. Mrak, M. Grgic, and M. Kunt, Eds., Signals and Communication Technology. Springer Berlin Heidelberg, 2010, 51-77. 